\title{
Monocyte chemoattractant protein-1 promotes macrophage-mediated tubular injury, but not glomerular injury, in nephrotoxic serum nephritis
}

\author{
Gregory H. Tesch, ${ }^{1}$ Andreas Schwarting, ${ }^{1}$ Koji Kinoshita, ${ }^{1}$ Hui Y. Lan, ${ }^{2}$ Barrett J. Rollins, ${ }^{3}$ \\ and Vicki Rubin Kelley ${ }^{1}$ \\ ${ }^{1}$ Laboratory of Molecular Autoimmune Disease, Renal Division, Brigham and Women's Hospital, Boston, Massachusetts 02115, USA \\ ${ }^{2}$ Department of Nephrology, Monash Medical Centre, Clayton, Victoria 3168, Australia \\ ${ }^{3}$ Department of Adult Oncology, Dana Farber Cancer Institute, Boston, Massachusetts 02115, USA
}

Address correspondence to: Vicki Rubin Kelley, Brigham and Women's Hospital, Harvard Institutes of Medicine, 77 Avenue Louis Pasteur, Boston, Massachusetts 02115, USA. Phone: (617) 525-5915; Fax: (617) 525-5830; E-mail: VKelley@rics.bwh.harvard.edu

Received for publication August 10, 1998, and accepted in revised form November 3, 1998.

\begin{abstract}
Monocyte chemoattractant protein-1 (MCP-1) is upregulated in renal parenchymal cells during kidney disease. To investigate whether MCP-1 promotes tubular and/or glomerular injury, we induced nephrotoxic serum nephritis (NSN) in MCP-1 genetically deficient mice. Mice were analyzed when tubules and glomeruli were severely damaged in the MCP-1-intact strain (day 7). MCP-1 transcripts increased fivefold in MCP-1-intact mice. MCP-1 was predominantly localized within cortical tubules (90\%), and most cortical tubules were damaged, whereas few glomerular cells expressed MCP-1 (10\%). By comparison, there was a marked reduction (>40\%) in tubular injury in MCP-1-deficient mice (histopathology, apoptosis). MCP-1-deficient mice were not protected from glomerular injury (histopathology, proteinuria, macrophage influx). Macrophage accumulation increased adjacent to tubules in MCP-1-intact mice compared with MCP-1-deficient mice $(70 \%, P<0.005)$, indicating that macrophages recruited by MCP1 induce tubular epithelial cell (TEC) damage. Lipopolysaccharide-activated bone marrow macrophages released molecules that induced TEC death that was not dependent on MCP-1 expression by macrophages or TEC. In conclusion, MCP-1 is predominantly expressed by TEC and not glomeruli, promotes TEC and not glomerular damage, and increases activated macrophages adjacent to TEC that damage TEC during NSN. Therefore, we suggest that blockage of TEC MCP-1 expression is a therapeutic strategy for some forms of kidney disease.
\end{abstract}

J. Clin. Invest. 103:73-80 (1999).

\section{Introduction}

The infiltration of monocytes and T cells into the kidney mediates tissue injury (1). During renal injury, infiltrating cells accumulate in glomeruli and in the interstitium, where their interaction with resident kidney cells, including tubular epithelial cells (TEC), stimulates the infiltrating and resident cells to generate molecules that promote kidney injury. Consequently, the accumulation of monocytes and $\mathrm{T}$ cells, and the ensuing inflammation, gives rise to glomerulosclerosis, crescent formation, tubular atrophy, interstitial fibrosis, and progressive renal failure. The infiltration of monocytes and $\mathrm{T}$ cells into the kidney is dependent on chemokines released by injured resident kidney cells $(1,2)$. These chemokines belong to a "superfamily" of small chemoattractant proteins that promote the recruitment and activation of infiltrating cells (3). During inflammation, the sustained release of chemokines by injured cells results in an increasing concentration gradient that is maximal within damaged tissue and is responsible for the unidirectional movement of infiltrating cells (2). Because chemokines are not crucial for most normal tissue functions $(4,5)$, and are induced during inflammation, they are attractive targets for therapeutic intervention for a broad array of diseases. In this group of chemokines, attention has focused on monocyte chemoattractant protein-1 (MCP-1) because it is a potent chemoattractant of monocytes, T cells, and natural killer cells (6-8). Consequently, blocking MCP-1 offers therapeutic possibilities in kidney diseases dependent on T-cell and/or monocyte-mediated injury, including IgA nephropathy, lupus nephritis, membranoproliferative glomerulonephritis, rapid progressive glomerulonephritis, and tubulointerstitial nephritis (9-12).

MCP-1 may be responsible for inducing kidney tubular and/or glomerular damage. Renal parenchymal cells (RPC), most notably mesangial cells and TEC, are known to produce MCP-1 in response to interleukin-1, tumor necrosis factor- $\alpha$, interferon- $\gamma$, and circulating IgG complexes (13-16). TEC and mesangial cells express MCP-1 during human and experimental kidney diseases $(9,12$, 17-22). Experimental studies have linked MCP-1 expression in glomeruli with an influx of macrophages using anti-thymocyte serum (16). Similarly, MCP-1 expression by TEC is noted to increase the number of macrophages in renal ischemia, ureteral obstruction, and protein overload (17-19). Because MCP-1 is among a vast array of molecules induced in glomeruli and TEC during inflammation (2), it is uncertain whether this chemokine contributes to glomerular and/or tubulointerstitial inflammation. 

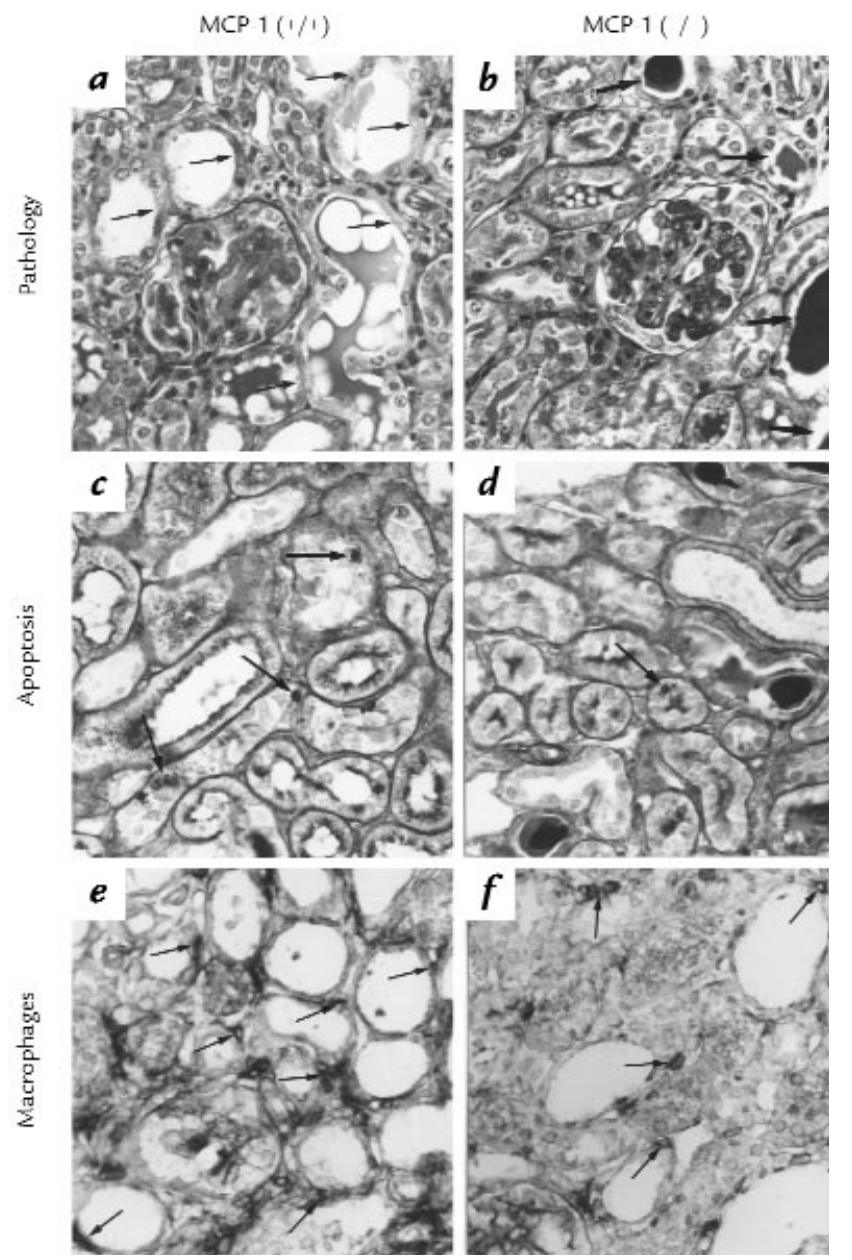

Figure 1

Histopathology and apoptosis are reduced in renal tubules in MCP$1(-/-)$ compared with MCP-1(+/+) mice given nephrotoxic serum. Cortical tubular injury was extensive in $\mathrm{MCP}-1(+/+)$ mice receiving nephrotoxic serum (a) (arrows; hematoxylin and PAS) but was reduced in MCP-1(-/-) mice (b). Glomerular injury was similar in both strains. More TEC were apoptotic in MCP-1(+/+) mice $(c)$ compared with MCP-1(-/-) mice $(\boldsymbol{d})$ (TUNEL; arrows). Many F4/80+ peritubular macrophages were detected in MCP- $1(+/+)$ mice $(\boldsymbol{e})$ (arrows) but were reduced in MCP-1(-/-) mice $(f) . \times 1000 . M C P-1$, monocyte chemoattractant protein-1; PAS, periodic acid-Schiff; TEC, tubular epithelial cells; TUNEL, terminal deoxynucleotide transferase-mediated dUTP nick end-labeling.

Gene knockout strains deficient in MCP-1 or its receptor, CCR2, have established the requirement for MCP-1 in inflammation. MCP-1 and CCR2-deficient mice have a reduction in macrophage recruitment and inflammation in thioglycolate-elicited peritonitis, delayed-type hypersensitivity, and pulmonary granulomas compared with their wild-type counterparts (5, 23-25). Therefore, MCP-1 contributes to inflammation, but it is uncertain whether it is limited to select tissues and recruits specific macrophages.

In this study, we tested the hypothesis that MCP-1 is responsible for glomerular and/or tubular kidney injury. To evaluate this hypothesis, we (a) induced glomerular and tubular injury in MCP-1-intact and -deficient mice using nephrotoxic serum, an experimental model con- sisting of an influx of monocytes and T cells resulting in glomerular and tubular injury, and $(b)$ identified the intrarenal pathogenic mechanisms dependent on MCP-1.

\section{Methods}

Mice. MCP-1-intact and -deficient B6/129 mice (129SV/J × C57BL/6) $F_{1}$ (provided by B.J. Rollins, Dana Farber Cancer Institute, Boston, Massachusetts, USA) were maintained in a pathogen-free animal facility. MCP-1-deficient mice were created by targeted gene disruption (5). Control MCP1 -intact mice were derived from matings of mice heterozygous for the disrupted allele.

Nephrotoxic serum nephritis (NSN). Nephrotoxic serum was prepared by immunizing sheep with a particulate fraction of mouse glomerular basement membrane from normal C57BL/6 mouse kidney (provided by H.Y. Lan, Monash Medical Centre, Clayton, Victoria, Australia) (26). Nephrotoxic serum was absorbed overnight against an equal volume of freshly prepared red blood cells from B6/129 mice. The serum supernatant was removed, heat-inactivated at $56^{\circ} \mathrm{C}$ for $30 \mathrm{~min}$, and dialyzed against PBS. The nephrotoxic serum was filtered $(0.2 \mu \mathrm{m})$, and the protein concentration was adjusted to $50 \mathrm{mg} / \mathrm{ml}$. Control serum was similarly prepared using pooled normal sheep serum (Sigma Chemical Co., St. Louis, Missouri, USA).

MCP-1-intact and MCP-1-deficient male mice (8 weeks of age, 22-25 g, n=6) were injected subcutaneously with $1 \mathrm{mg}$ of sheep IgG mixed with CFA (1:1) (Sigma Chemical Co.). To avoid super acute hypersensitivity caused by the administration of sheep anti-mouse nephrotoxic serum, these mice were challenged 5 days later with intravenous injections of $50 \mu \mathrm{l}(2.5 \mathrm{mg})$ of either nephrotoxic serum or control serum for 3 consecutive days. Mice were sacrificed on day 7 .

MCP-1 transcripts. Identification of the disrupted and nondisrupted MCP-1 genes in experimental mice was determined by PCR analysis. DNA was extracted from mouse tails using the QIAmp tissue kit (QIAGEN GmbH, Hilden, Germany). The DNA was assessed by PCR using oligonucleotide primers that recognize either the normal MCP-1 gene (antisense R2: 5' ACA GCT TCT TTG GGA CAC C 3', sense F1: 5' GGA GCA TCC ACG TGT TGG C $3^{\prime}$ ) or the mutant MCP-1 gene containing the target disruption (antisense R3: 5' ACG ATG TCG TCG TGA CCC ATG GCG A 3', sense F1). Gel analysis of the PCR products identified the normal MCP-1 gene fragment at $800 \mathrm{bp}$ and the MCP-1 mutant gene fragment at $1500 \mathrm{bp}$.

Western blot analysis of MCP-1 expression. Bone marrow macrophages and glomeruli were extracted $(27,28)$ from MCP1 -intact and -deficient mouse strains at day 7 of NSN. Macrophages $\left(10^{6} / \mathrm{ml}\right)$, glomeruli $\left(2 \times 10^{3} / \mathrm{ml}\right)$, and mesangial cells $\left(10^{6} / \mathrm{ml}\right)$, were cultured in $10 \% \mathrm{FCS} / \mathrm{DME} \pm$ LPS $(5$ $\mu \mathrm{g} / \mathrm{ml}$ ) for $24 \mathrm{~h}$. Culture supernatants were collected and incubated overnight at $4^{\circ} \mathrm{C}$ with $5 \mu \mathrm{g} / \mathrm{ml} \mathrm{rabbit} \mathrm{anti-mouse} \mathrm{MCP-}$ 1 antibody (Serotec Ltd., Oxford, United Kingdom). The supernatant was then incubated for a further $2 \mathrm{~h}$ at $4^{\circ} \mathrm{C}$ with goat anti-rabbit IgG conjugated to magnetic beads (BioMag; PerSeptive Biosystems Inc., Framingham, Massachusetts, USA). The magnetic beads were collected, washed, and incubated at $95^{\circ} \mathrm{C}$ for $5 \mathrm{~min}$ in nonreducing SDS-PAGE buffer. The samples were run on a $12 \%$ acrylamide gel. MCP- 1 was determined by Western blotting. Blots were preincubated in 5\% nonfat milk, 0.1\% Tween-20, $150 \mathrm{mM} \mathrm{NaCl}, 50 \mathrm{mM}$ Tris- $\mathrm{HCl}$ $(\mathrm{pH} 7.5)$ to prevent nonspecific antibody binding, and then incubated with rabbit anti-mouse MCP-1 IgG (1:5,000; Serotec Ltd.) followed by goat anti-rabbit IgG conjugated with horse radish peroxidase (1:10,000; Sigma Chemical Co.). The blots were developed using ECL Supersignal reagent (Pierce Chemical Co., Rockford, Illinois, USA), and the resulting signal was captured on Kodak X-OMAT film. 
$\boldsymbol{a}$

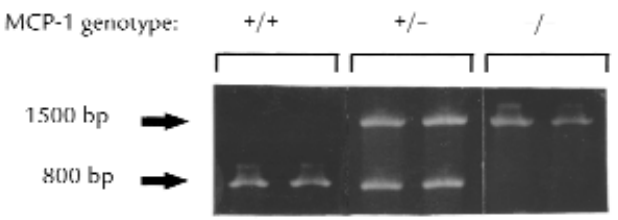

b

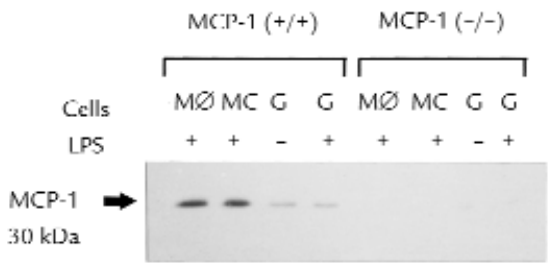

Figure 2

MCP-1-intact mice have nondisrupted MCP-1 DNA and express MCP-1 protein. (a) Fragments of genomic MCP-1 (800 bP) and the mutated MCP-1 gene (1500 bp) were detected by PCR in DNA extracted from the tails of B6/129 mice. Two samples from MCP-1-homozygous (+/+), -heterozygous (+/-), and -deficient (-/-) mice are represented. (b) MCP-1 protein is expressed by bone marrow macrophages $(M \varnothing)$, mesangial cells $(M C)$, and glomeruli $(G)$ in MCP-1(+/+) mice but is absent in deficient $(-/-)$ mice. Cells and glomeruli were extracted at day 7 of NSN and cultured for $24 \mathrm{~h}$ in medium alone or medium with $5 \mu \mathrm{g} / \mathrm{ml}$ LPS. The culture supernatants were analyzed for the presence of MCP-1 by Western blotting. NSN, nephrotoxic serum nephritis.

Proteinuria. Urine protein levels in mice receiving nephrotoxic or control serum were assessed semi-quantitively by dipstick analysis (Albustix; Bayer Diagnostics Division, Elkhart, Indiana, USA), beginning before injection and on days 1, 2, 3, 5 , and 7 after the initial challenge injection.

Renal pathology. Kidney sections were fixed in $10 \%$ formalin for $24 \mathrm{~h}$ at $4^{\circ} \mathrm{C}$. Paraffin sections $(4 \mu \mathrm{m})$ were stained with hematoxylin and periodic acid-Schiff reagent (PAS). Glomerular hypercellularity was assessed by counting the number of nucleated cells in 20 glomerular cross-sections (gcs) per kidney. We evaluated glomerular pathology by assessing 100 ges per kidney and determined the percentage of glomeruli with crescents (defined as two or more cell layers within Bowman's space) and segmental lesions (exhibiting at least one of the following: necrosis, proliferation, hyalinosis). The percentage of damaged tubules (consisting of at least one of the following: dilation, atrophy, necrosis) was determined by scoring 400 renal cortical tubules per kidney in randomly selected microscopic fields (400×). All scoring was performed on blinded slides.

Antibodies. The following primary antibodies were used for immunostaining: rat anti-mouse CD4 IgG2a clone RM4-5 (PharMingen, San Diego, California, USA) to detect CD4 T cells; rat anti-mouse CD8a (Ly-2) IgG2a clone 53-6.7 (PharMingen) to detect CD8 T cells; rat anti-mouse monocyte/macrophage IgG2b clone MOMA-2 (BioSource International Inc., Camarillo, California, USA) and rat anti-mouse macrophage IgG2b (prepared from F4/80 hybridoma supernatant, ATCC No. HB198) to detect macrophages; rabbit anti-mouse MCP-1 IgG (Serotec Ltd.) to detect MCP-1; fluorescein-conjugated rabbit anti-sheep IgG (Organon Teknika Corp., West Chester, Pennsylvania, USA) to detect sheep IgG deposits; fluorescein-conjugated goat anti-mouse IgG (Organon Teknika Corp.) to detect mouse IgG deposits; fluorescein-conjugated goat anti-mouse C3 (Organon Teknika Corp.) to detect mouse complement C3 deposits; and mouse anti-cytokeratin peptide 18 IgG1 clone CY90 (Sigma Chemical Co.) to detect epithelial cells. The negative isotype control antibodies were rat IgG2a clone R35-95, rat
IgG2b clone R35-38, mouse IgG1 clone MOPC-21 (PharMingen), and normal rabbit IgG (Sigma Chemical Co.). The secondary antibodies for immunostaining were biotin-conjugated rabbit anti-rat IgG and biotin-conjugated goat anti-rabbit IgG (Vector Laboratories, Burlingame, California, USA).

Assessment of IgG and C3 deposition in the kidney. To examine IgG and $\mathrm{C} 3$ deposits in the kidney, cryostat tissue sections $(4 \mu \mathrm{m})$ were incubated with $20 \%$ normal goat or rabbit serum (30 min) and then incubated with fluorescein-conjugated antibodies detecting sheep IgG, mouse IgG, or mouse C3 (30 min). Tissue sections were washed and mounted with VECTASHIELD (Vector Laboratories). Immunofluorescence staining was assessed by titrating the antibodies on serial tissue sections, using twofold dilution steps (1:100-1:25,600), and determining the dilution at which specific staining disappears by fluorescence microscopy. Identification of kidney-infiltrating cells by immunostaining. To analyze kidney-infiltrating $\mathrm{T}$ cells and interstitial macrophages, cryostat tissue sections $(5 \mu \mathrm{m})$ were fixed in ethanol at $4^{\circ} \mathrm{C}$ for $10 \mathrm{~min}$ and immunostained with CD4, CD8, and F4/80 antibody as described previously (27), using an avidinbiotin-peroxidase detection system (Vector Laboratories). Glomerular macrophages were identified in $4-\mu \mathrm{m}$ acetone-fixed tissue sections using MOMA-2 antibody as reported previously (29). Interstitial cell infiltration was evaluated by counting the number of immunostained cells in the interstitium in 10 randomly selected microscopic fields $\left(100 \mu \mathrm{m}^{2}\right)$ per kidney. Glomerular and periglomerular cell accumulation was assessed by counting the number of labeled cells within or adjacent to each glomeruli, scoring 20 glomeruli at random per cross-section. Perivascular cell accumulation was determined by semiquantitative scoring of cells surrounding 10 random inter- and intralobular arteries (score: $0=$ none; $1=<2$ cell layers surrounding at least half the vessel; $2=2-5$ cell layers surrounding at least half the vessel; $3=>5$ cell layers surrounding at least half the vessel). Peritubular cell accumulation was determined by counting the number of labeled cells adjacent to a tubule. We calculated the mean of 400 tubules per section selected at random. Accumulation of macrophages and $\mathrm{T}$ cells within glomeruli, adjacent to glomeruli, or adjacent to cortical tubules was expressed as a cell index based on the number of glomeruli and cortical tubules per section. In addition, peritubular macrophage accumulation was correlated with the tubular damage score obtained from the same animal.

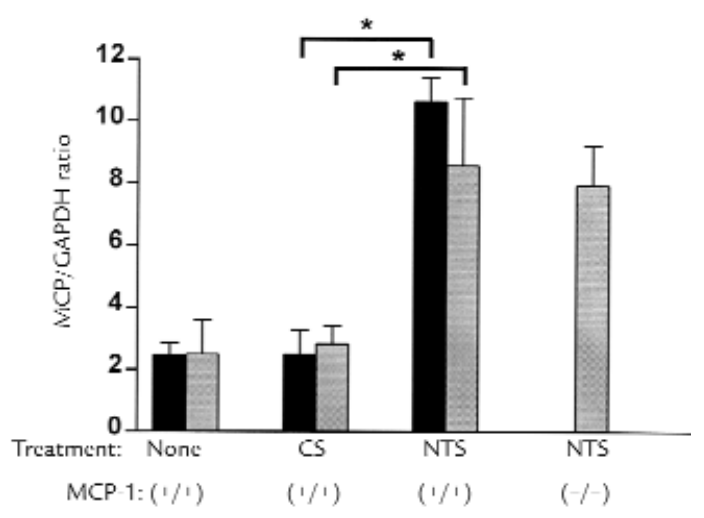

Figure 3

Renal transcripts of MCP-1 and MCP-3 increase in the renal cortex of MCP-1 (+/+) mice given nephrotoxic serum. Semi-quantitative PCR analysis was used to determine MCP-1 (black bars) and MCP-3 (gray bars) in the renal cortex. Mice received either nephrotoxic serum (NTS), control serum (CS), or were not treated (None). Mice were sacrificed 7 days after treatment. Mean $\pm \mathrm{SD}, n=6,{ }^{*} P<0.0001$. GAPDH, glyceraldehyde3-phosphate dehydrogenase. 


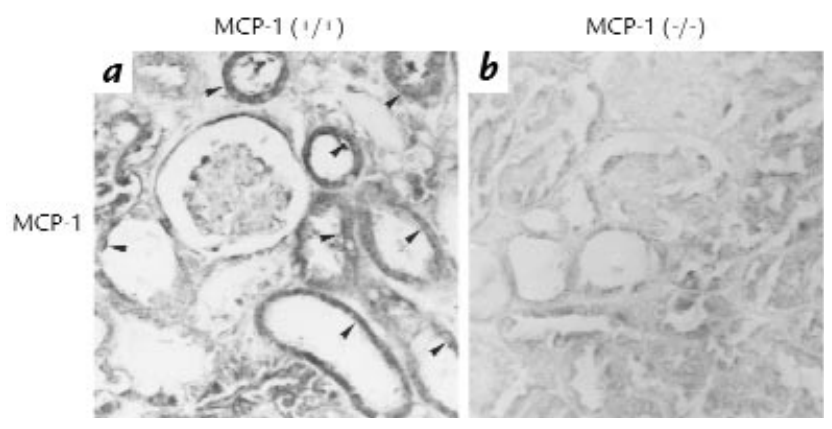

Figure 4

MCP-1 is predominantly expressed in injured tubules in NSN. MCP-1 was strongly expressed by most TEC but was detectable in only a few glomerular cells in MCP-1(+/+) mice at day 7 after receiving nephrotoxic serum (a) (arrowheads); it was absent in MCP-1(-/-) mice (b).

Identification of MCP-1 by immunostaining. To detect MCP-1, formalin-fixed tissue sections $(5 \mu \mathrm{m})$ were deparaffinized and incubated with $20 \%$ normal goat serum for $30 \mathrm{~min}$. Tissue sections were then incubated with MCP-1 antibody $(10 \mu \mathrm{g} / \mathrm{ml})$ in $1 \%$ BSA overnight at $4^{\circ} \mathrm{C}$. Bound primary antibody was labeled with biotin-conjugated goat anti-rabbit IgG for $1 \mathrm{~h}$ and subsequently detected using the avidin-biotin-peroxidase system (Vector Laboratories). Glomerular MCP-1 was assessed as described for glomerular- infiltrating cells. Because MCP1 was not detected in medullary tubules (data not shown), tubular MCP-1 was determined by counting the number of cortical tubules with TEC expressing MCP-1, scoring 400 tubules per cross-section.

Identification of apoptotic cells in the kidney. Apoptotic cells in kidney sections were identified by the terminal deoxynucleotide transferase-mediated dUTP nick end-labeling (TUNEL) method and immunoperoxidase staining (In Situ Cell Death Detection Kit; Boehringer Mannheim Indianapolis, Indiana, USA) Apoptosis in TEC was assessed by counting TUNEL-labeled cells in 500 cortical tubules. TUNEL labeling and immunostaining was scored using coded slides.

PCR assessment of renal mRNA. Total RNA was extracted from the snap-frozen renal cortex of half a kidney using RNAzol B (Tel-Test Inc., Friendswood, Texas, USA). A reverse transcription (RT) reaction was performed on this RNA using oligo-dT and the Superscript II DNA preamplification kit (GIBCO BRL, Grand Island, New York, USA). The resulting RT product was used as a cDNA template for PCR analysis. Glyceraldehyde-3-phosphate dehydrogenase (GAPDH) expression was detected as a 500-bp product resulting from PCR with specific oligonucleotide primers (antisense: $5^{\prime}$ CAA AGT TGT CAT GGA TGA CC 3', sense: $5^{\prime}$ GGT GGA GGT CGG AGT CAA CG). Similarly, MCP-1, MCP-3, and MCP-5 expression were detected as 350-bp, 300-bp, and 550-bp PCR products, respectively, using specific oligonucleotide primers (MCP-1 antisense: 5' GCTTGAGGTGGTTGTGGAAAA 3', MCP-1 sense: $5^{\prime}$ CTCACCTGCTGCTACTCATTC 3'; MCP-3 antisense: 5' CACATTCCTACAGACAGCTC 3', MCP-3 sense: $5^{\prime}$ AGCTACAGAAGGATCACCAG 3'; MCP-5 antisense: 5' CTCCTTATCCAGTATGGTCC $3^{\prime}$, MCP-5 sense: $5^{\prime}$ TCTCCTCCACCATGCAGAG 3').

Cell culture assays: analysis of TEC death. Bone marrow macrophages (BMM $\varnothing)$ and TEC were extracted from MCP1-intact and -deficient mice, characterized by morphology and antibody staining (anti-macrophage F4/80 antibody, anti-epithelial cell CY-90 antibody), and grown in culture as reported previously $(27,30)$. We established whether MCP-1 was responsible for inducing TEC death. MCP-1-intact and -deficient BMM $\varnothing$ $\left(10^{6} / \mathrm{ml}\right)$ were incubated for $12-48 \mathrm{~h}$ in medium alone (K1 medi- um without epidermal growth factor), or with medium containing rmMCP-1 (100 ng/ml; PharMingen) or LPS ( $5 \mu \mathrm{g} / \mathrm{ml}$; Sigma Chemical Co.). The supernatant from these BMM $\varnothing$ was collected and added ( $1 \mathrm{ml} /$ well) to TEC $\left(10^{5} /\right.$ well $)$ grown in 24 -well plates. TEC were cultured for $48 \mathrm{~h}$ with BMM $\varnothing$ supernatants, media alone, or media containing rmMCP- 1 or LPS. TEC necrosis was measured by removing cells with trypsin, staining cell suspensions with $0.1 \%$ trypan blue, and counting the cells using a hemacytometer. Samples of 150-200 cells were assessed in triplicate and expressed as a percentage of necrotic TEC. These experiments were repeated three times using cells extracted from additional mice. TEC death by apoptosis and necrosis was determined by staining with $1 \mu \mathrm{g} / \mathrm{ml}$ propidium iodide (Sigma Chemical Co.) and annexin V (Boehringer Mannheim) as reported previously (31). Apoptotic TEC were labeled with annexin $V$ but were impermeable to propidium iodide, whereas necrotic TEC were stained with propidium iodide.

Statistics. Data was analyzed using the Mann-Whitney Wilcoxon test and Spearman's correlation coefficient.

\section{Results}

Morphological assessment of renal injury in MCP-1 intact mice induced by NSN. Renal tubular and glomerular damage in NSN is severe by seven days. Renal pathology was limited to the cortex and did not involve the medulla. The majority of cortical tubules $(51 \% \pm 5 \%)$ were damaged at day 7 of NSN. Tubular damage included dilation with flattened or degraded epithelium, atrophy, and thickening or rupture of basement membranes (Fig. 1a). Peritubular infiltrating cells and protein casts were also associated with most damaged tubules. Glomerular injury was extensive (97 \pm $2 \%$ ) and involved at least one of the following: hyalinosis, capillary dilation and thickening, focal segmental sclerosis, and crescents (Fig. 1a). Only a few glomeruli were totally obliterated $(<2 \%)$. We noted a prominent cell infiltrate around most glomeruli; however, we did not detect glomerular hypertrophy or hypercellularity.

Increased expression of monocyte chemoattractant proteins in NSN. We verified that MCP-1-deficient mice did not express this chemokine, because (a) we identified only

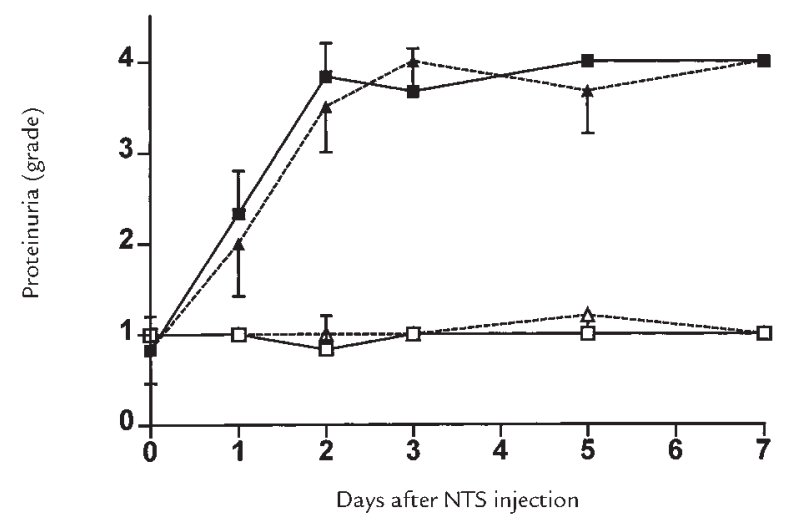

\section{Figure 5}

Proteinuria does not decrease in MCP-1(-/-) mice given NTS. Urine protein excretion from MCP-1(+/+) mice (solid line) and MCP-1(-/-) mice (dotted line) was measured after the administration of either NTS (filled symbols) or control serum (unfilled symbols), and scored on a semi-quantitative scale. Mean $\pm \mathrm{SD}, n=6, \mathrm{MCP}-1(+/+)$ vs. MCP-1(-/-) mice $(P>$ $0.2)$. NTS, nephrotoxic serum. 
the disrupted MCP-1 gene in the genomic DNA of MCP-1-deficient mice using PCR, whereas MCP1 -intact mice had at least one allele of the nondisrupted MCP-1 gene (Fig. 2a), and (b) MCP-1 was not detected in lipopolysaccharide (LPS)-stimulated BMM $\varnothing$ and RPC supernatants from MCP-1-deficient mice compared with MCP-1-intact mice using Western blot analysis (Fig. 2b).

MCP-1 was upregulated in the kidney in NSN. There was an increase in MCP-1 transcripts (four- to fivefold) in the renal cortex of MCP-1-intact mice at day 7 of NSN compared with MCP-1-intact mice receiving control sera or uninjected controls (Fig. 3). MCP-1 was identified predominantly in cortical tubules $(>90 \%)$ in mice with NSN by immunostaining (Fig. 4a). Lesser amounts of MCP-1 were expressed in glomerular crescents, parietal epithelial cells, and a small proportion of cells $(10 \%)$ within the glomerular tuft $(4.2 \pm 0.3$ cells/gcs) during NSN. Thus, MCP-1 expression in TEC far exceeds the amount within glomeruli.

Because MCP-3 and MCP-5 are also ligands for the MCP-1 receptor (CCR2), we examined whether kidney expression of these molecules is upregulated following renal injury. Constitutive mRNA expression of MCP-3 increased three- to fourfold in MCP-1-intact and -deficient mice with NSN as measured by RT-PCR (Fig. 3). In contrast, MCP-5 was not detected in the kidney of MCP1 -intact or -deficient strains. We ensured that MCP-3 and MCP-5 could be detected by establishing the presence of these molecules in the spleens of MCP-1-intact and-deficient mice (data not shown).

The absence of MCP-1 does not prevent $I g G / C 3$ deposition and proteinuria in NSN. Sheep IgG, mouse IgG, and complement C3 were prominent on the glomerular basement membrane in mice that received nephrotoxic serum. The localization and level of IgG (sheep and mouse) and C3 deposition was not different in MCP1-intact and-deficient mice, as determined by antibody titration (data not shown).

We did not detect any difference in the incidence or severity of proteinuria in MCP-1-intact and -deficient mice receiving nephrotoxic serum (Fig. 5). MCP-1-intact mice given nephrotoxic serum became rapidly proteinuric. Proteinuria was evident at day 1 of disease and peaked at day 3 ; it remained elevated until the mice were sacrificed on day 7 after receiving nephrotoxic serum. By comparison, MCP-1-intact and-deficient mice receiving control sera remained nonproteinuric.
The absence of MCP-1 protects from damage to tubules, but not glomeruli, in NSN. We detected fewer damaged cortical tubules in MCP-1-deficient compared with MCP1-intact strains with NSN using histopathologic criteria (Fig. $6 a$ and Fig. 1, $a$ and $b$ ). This was associated with a reduction in the percentage of apoptotic tubules (Fig. $6 b$ and Fig. 1, $c$ and $d$ ). In contrast, MCP1 -deficient glomeruli were not protected from renal injury. There was no difference in the number of damaged glomeruli (97 $\pm 2 \%$ ), the severity of damage, or crescent formation $(12 \pm 2 \%)$ in the MCP-1-intact and -deficient strains with NSN.

The absence of MCP-1 reduces the accumulation of macrophages in the interstitium in NSN. F4/80 antibody detected macrophages in periglomerular, peritubular, and perivascular lesions but did not stain for the presence of macrophages within glomeruli in MCP-1-intact and -deficient mice. In contrast, MOMA-2 antibody detected macrophages within glomeruli and labeled fewer macrophages in the interstitium compared with F4/80. Therefore, we analyzed macrophages in the interstitium and glomeruli with F4/80 antibody and MOMA-2 antibody, respectively.

At day 7 of NSN, the number of peritubular macrophages correlated with the extent of damage in cortical tubules $(r=0.93, P<0.0001, n=12)$ in MCP-1-intact and -deficient mice. There were fewer macrophages in the interstitium and adjacent to damaged tubules in MCP-1deficient kidneys compared with MCP-1-intact kidneys (Fig.7 and Fig. 1, $e$ and $f$ ). In contrast, we detected no difference in the number of macrophages within glomeruli (Fig. 7), nor in the glomerular cell number ( $40 \pm 4$ cells $\mathrm{MCP}-1(+/+)$ vs. $43 \pm 2$ cells MCP-1(-/-), $P=0.2)$ in MCP1 -intact and-deficient mice.

Infiltrating $\mathrm{CD}^{+}$and $\mathrm{CD}^{+} \mathrm{T}$ cells were seen mainly in perivascular areas but were also seen adjacent to glomeruli and tubules, and within glomeruli (Fig. 7). There was no difference in T-cell accumulation in MCP1-intact and -deficient strains (Fig. 7; perivascular CD4 cell score: $0.9 \pm 0.3 \mathrm{MCP}-1(+/+)$ vs. $0.8 \pm 0.2 \mathrm{MCP}-1(-/-)$, $P=0.2$; perivascular CD8 cell score: $0.3 \pm 0.2 \mathrm{MCP}-1(+/+)$ vs. $0.2 \pm 0.2 \mathrm{MCP}-1(-/-), P=0.3)$.

MCP-1 does not induce TEC death. Because tubule damage is reduced in MCP-1-deficient mice given nephrotoxic serum, we examined whether MCP-1 induced TEC death. rMCP-1 $(100 \mathrm{ng} / \mathrm{ml})$ did not reduce the viability of MCP-1-intact or -deficient TEC in culture (Fig. 8). Therefore, MCP-1 does not directly destroy TEC.
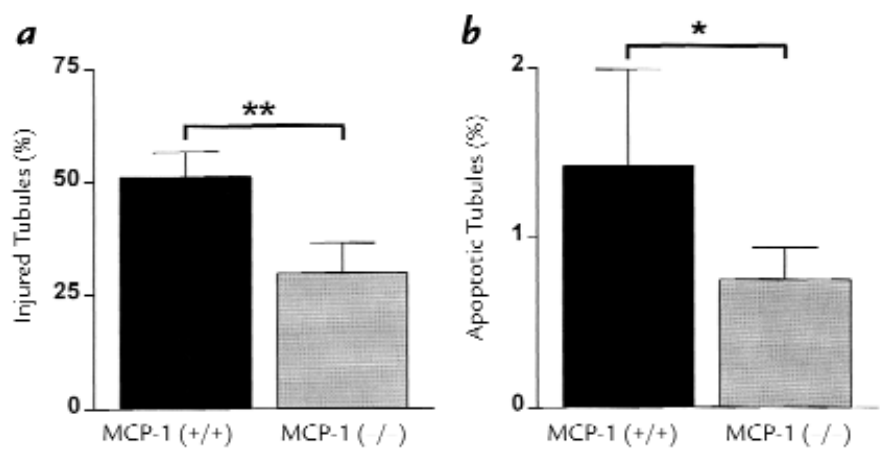

Figure 6
Reduced renal tubule damage in MCP- $1(-/-)$ mice given nephro-
toxic serum. Tubule injury was assessed at day 7 of NSN by $(\boldsymbol{a})$
histology (hematoxylin and PAS) and $(\boldsymbol{b})$ TUNEL labeling of apop-
totic TEC. Tubules $(400)$ were scored in the renal cortex in ran-
domly selected fields $(\times 400)$. Black bars represent MCP-1 $(+/+)$
and gray bars represent MCP-1(-/-) mice. Mean $\pm \mathrm{SD}, n=6,{ }^{*} P$
$<0.05,{ }^{*} P<0.0005$.

The Journal of Clinical Investigation ｜ January 1999 | Volume $103 \quad$ Number 1 


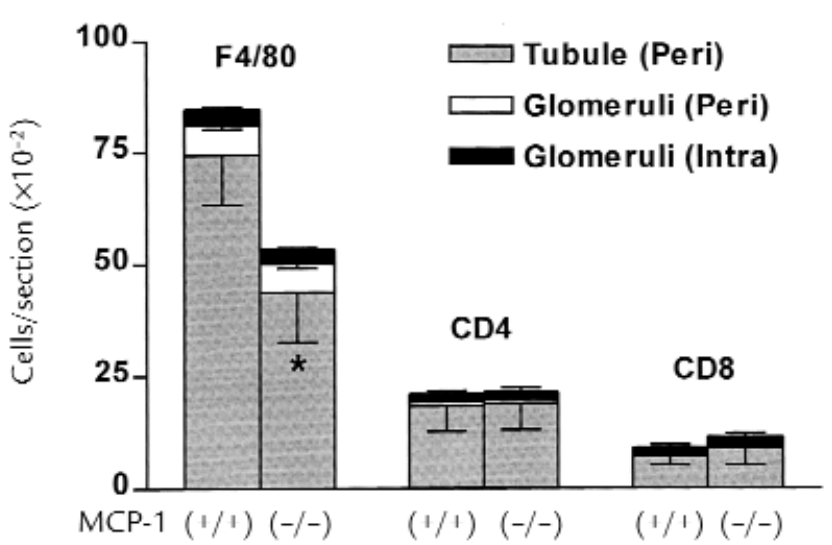

Figure 7

Peritubular macrophages, but not $\mathrm{T}$ cells or glomerular macrophages, are reduced in MCP-1(-/-) mice given nephrotoxic serum. Intrarenal macrophages and T cells were assessed in MCP- $1(+/+)$ and MCP-1(-/-) mice 7 days after receiving nephrotoxic serum. Macrophages were detected with F4/80 antibody (interstitium, periglomerular) and MOMA-2 antibody (intraglomerular). Interstitial and glomerular $T$ cells were detected using CD4 and CD8 antibodies (scoring: periglomerular cells/glomerular cross-section (gcs); peritubular cells/tubule cross-section; intraglomerular cells/gcs). Mean $\pm \mathrm{SD}, n=6,{ }^{*} P<0.005$ compared with MCP-1(+/+).

Macrophages release molecules that induce TEC apoptosis. To test the hypothesis that activated macrophages induce TEC damage, we incubated TEC with supernatant derived from activated BMMØ. Supernatant from LPS-stimulated BMMØ induced greater TEC death (16\%-24\%) compared with unstimulated BMMØ (5\%-7\%), whereas TEC death induced by MCP-1-stimulated BMM $\varnothing$ did not differ from unstimulated BMM (Fig. 8). TEC death was not dependent on whether BMMØ or TEC were derived from MCP1-intact or-deficient mice, and LPS was not directly toxic to TEC. Furthermore, coculture of BMM $\varnothing$ with TEC in the presence of LPS induced TEC death similar to BMM $\varnothing$ supernatant alone (data not shown). We determined that macrophage-mediated TEC cell death was in part due to apoptosis, because a proportion of TEC $(18 \% \pm 5 \%)$ that were not necrotic (TEC impermeable to propidium iodide) stained with the apoptosis marker, annexin $\mathrm{V}$, after treatment with LPS-stimulated BMM $\varnothing$ supernatant.

\section{Discussion}

In this report, we tested the hypothesis that MCP-1 is required for tubular or glomerular damage in an induced model of renal injury, NSN. We report that in NSN, MCP-1 increases predominantly in TEC in the kidney and is sparse within glomeruli. Furthermore, macrophage accumulation adjacent to TEC correlates with tubular damage and increased tubular MCP-1 expression. We conclude that MCP-1 recruits macrophages that are already activated, or become activated within the kidney, and release molecules responsible for TEC death.

The correlation of peritubular macrophages, MCP-1 expression on TEC, and tubular damage suggests that MCP-1-mediated tubule damage is dependent on macrophages. This concept is supported by our findings that (a) macrophage accumulation adjacent to tubules in NSN is reduced in MCP-1-deficient mice, and (b) activated BMMØ release molecules that cause TEC death. Our findings also suggest that MCP-1 recruits, but does not stimulate, macrophages to release molecules that induce TEC death. This is consistent with reports that transgenic mice expressing MCP- 1 in alveolar epithelial cells or pancreatic islet cells induce local macrophage accumulation but require an additional signal (LPS) for inflammation to ensue $(32,33)$. In our study, we established that MCP-1 does not induce macrophages to release molecules toxic to tubules. It will be important to determine whether macrophages recruited by MCP- 1 expressed on TEC are stimulated to become nephrotoxic within the kidney.

TEC death is not exclusively dependent on MCP1 -recruited macrophages. In the absence of MCP-1, macrophage accumulation and tubular damage are reduced, but not eliminated, in NSN. In addition, although MCP-1 is induced in TEC in another model of kidney injury, unilateral ureteral ligation (UUL), MCP1-deficient kidneys are not protected from an influx of macrophages or tubular damage (our unpublished findings) One possible explanation is that the mechanical induction of tubular damage in UUL induces an array of additional molecules that are responsible for recruiting macrophages. Thus, MCP- 1 is made redundant by other macrophage-recruiting molecules. By comparison, although immune-mediated renal injury in NSN is not entirely dependent on MCP-1 to recruit macrophages, MCP-1 is responsible for a large proportion (40\%) of these tubular damaging cells.

There are several reasons that would explain why the glomerular injury was not protected in the absence of MCP-1 in NSN. First, renal injury may require a threshold of MCP-1 that was not reached in glomeruli. Cortical TEC expression of MCP-1 is abundant in MCP-

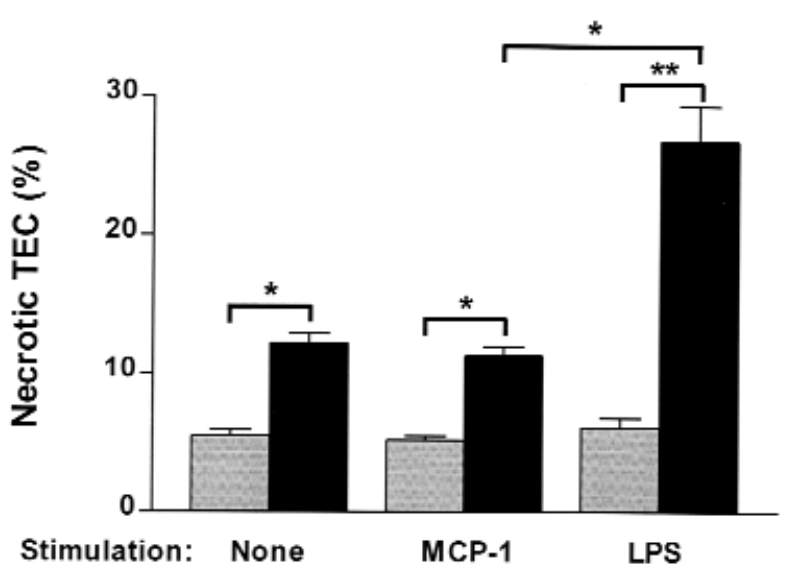

\section{Figure 8}

TEC are injured by MØSN. TEC were cultured in medium with and without stimuli (gray bars), or in the presence of MØSN (black bars). TEC from MCP-1(+/+) mice were cultured with M $\varnothing \mathrm{SN}$ from MCP-1(+/+) mice. After incubation, cells were briefly trypsinized and stained with $0.1 \%$ trypan blue. The percentage of necrotic TEC was determined by counting trypan blue-stained cells. Results are based on the mean values from three experiments. Similar results were obtained using TEC and M $\varnothing S N$ from MCP-1 (-/-) mice (data not shown). Mean $\pm \mathrm{SD}, n=3,{ }^{*} P<0.01$, ${ }^{*} P<0.005 . M \varnothing S N$, macrophage supernatant. 
1-intact mice with NSN, whereas glomerular expression is sparse. Thus, it is not surprising that the impact of removing MCP-1 from glomeruli is negligible. Second, glomerular deposition of IgG and C3 was the same in MCP-1-intact and-deficient mice that received nephrotoxic serum. Therefore, the recruitment of glomerular macrophages via Fc- and C3-dependent mechanisms is unlikely to be different in MCP-1-deficient mice with NSN. Third, macrophages recruited to the tubules and glomeruli by MCP-1 may differ. We noted that the macrophages in the interstitium and glomeruli expressed different antigenic markers (34). These macrophage antigen markers may reflect distinct states of maturation, and in turn, responsiveness to MCP-1 recruitment. Fourth, chemokines other than MCP-1 released by glomerular cells during NSN may be responsible for recruiting of macrophages. Glomeruli isolated from rats with NSN have an increase in CC chemokine transcripts that attract macrophages, including macrophage inflammatory protein (MIP)- $1 \alpha$, MIP- $1 \beta$, RANTES, TCA3, and MCP-3 (35). Furthermore, other CC chemokines associated with macrophage-dependent inflammation, including MCP-2, MCP-4, I309, and hemofiltrate CC. chemokine(HCC)-1 (3), may be released during glomerular injury. To add to this complexity, there are at least three monocyte $\mathrm{CC}$ chemokine receptors (CCR1, CCR2, and CCR5) (3). MCP-1 attracts macrophages by signaling through the CCR2 chemokine receptor, and in addition to MCP-1, MCP-3 and MCP-5 are coligands for this receptor $(36,37)$. Therefore, because intrarenal MCP-3, but not MCP-5, transcripts increase in MCP-1-intact and -deficient mice with NSN, MCP-3 may compensate for the lack of glomerular MCP1 signaling through CCR2. In addition, it is possible that B6/129 hybrids have undergone sorting for an allele(s) that provides protection against renal injury. However, this is unlikely because the extent of tubular damage correlated with the influx of macrophages adjacent to tubules. Clearly, the differential impact of MCP-1 on tubule, but not glomerular, injury has begotten a series of questions that will be instrumental in our understanding of the pathogenesis of kidney disease.

Genetic deletion of MCP-1 and blockade of MCP-1 via antibodies have a differing impact on kidney injury in NSN. Provision of MCP-1 antibody reduces glomerular injury in NSN (29, 38-40). In contrast, MCP-1-deficient mice given nephrotoxic serum were not protected from glomerular damage. These differences may be related to the amount, location, and period in which MCP-1 is eliminated during the development of renal disease. This is not surprising. In addition, there are numerous examples highlighting the differences between genetic targeted disruption and antibody strategies that target molecules promoting inflammation to thwart the progression of immune-mediated diseases (41-43). It is also worth noting that the differences in NSN are dependent on the preparation of nephrotoxic serum and on the animal strain. In fact, there is varying efficacy using anti-MCP-1 antibody in NSN; while Lloyd et al. provide evidence of glomerular protection at day 7 (29), another report claims that protection is transient and is lost after day 3 (40). These findings underscore the com- plexity of designing therapeutic strategies for eliminating MCP-1 to combat human kidney disease.

In conclusion, our data suggest that strategies that eliminate MCP-1 would protect against progressive loss of tubules in patients with tubulointerstitial kidney diseases. We are currently investigating the impact of eliminating MCP-1 in MRL-Fas ${ }^{l p r}$ mice that spontaneously develop glomerular and tubular injury closely resembling human lupus nephritis.

\section{Acknowledgments}

We wish to thank Clare M. Lloyd (Millenium Pharmaceuticals, Cambridge, Massacusetts, USA) for editorial assistance in the preparation of this manuscript. This work was supported in part by a National Kidney Foundation Fellowship Grant to G.H. Tesch, the Polycystic Kidney Research Foundation and the National Institute of Health and Grant DK 36149.

1. Paterson, D.J., Lan, H.Y., and Atkins, R.C. 1997. Macrophages in immune renal injury. In Immunologic renal disease. E.G. Neilson and W.G. Couser, editors. Raven Press. New York, NY. 575-592.

2. Rovin, B.H., and Phan, L.T. 1998. Chemotactic factors and renal inflammation. Am. J. Kidney Dis. 31:1065-1084.

3. Rollins, B.J. 1997. Chemokines. Blood. 90:909-928.

4. Cook, D.N., et al. 1995. Requirement for MIP- $1 \alpha$ for an inflammatory response to viral infection. Science. 269:1583-1585.

5. Lu, B., et al. 1998. Abnormalities in monocyte recruitment and cytokine expression in MCP-1 deficient mice. J. Exp. Med. 187:601-608.

6.Valente, A.J., Graves, D.T., Vialle-Valentin, C.E., Delgado, R., and Schwartz, C.J. 1988. Purification of monocyte chemotactic factor secreted by nonhuman primate vascular cells in culture. Biochemistry. 27:4162-4168.

7. Carr, M.W., Roth, S.J., Luther, E., Rose, S.S., and Springer, T.A. 1994. Monocyte chemoattractant protein-1 acts as a T-lymphocyte chemoattractant. Proc. Natl. Acad. Sci. USA. 91:3652-3656.

8. Allavena, P., et al. 1994. Induction of natural killer cell migration by monocyte chemotactic protein-1, -2 and -3 . Eur. J. Immunol. 24:3233-3236.

9. Rovin, B.H., Rumancik, M., Tan, L., and Dickerson, J. 1994. Glomerular expression of monocyte chemoattractant protein-1 in experimental and human glomerulonephritis. Lab. Invest. 71: 536-542.

10. Prodjosudjadi, W., et al. 1995. Production and cytokine-mediated regulation of monocyte chemoattractant protein-1 by human proximal tubular epithelial cells. Kidney Int. 48:1477-1486.

11. Wada, T., et al. 1996. Monitoring urinary levels of monocyte chemotactic and activating factor reflects disease activity of lupus nephritis. Kidney Int. 49:761-767.

12. Grandaliano, G., et al. 1996. Monocyte chemotactic peptide-1 expression in acute and chronic human nephritides: a pathogenetic role in interstitial monocytes recruitment. J. Am. Soc. Nephrol. 7:906-913.

13. Rovin, B.H., Yoshiumura, T., and Tan, L. 1992. Cytokine-induced production of monocyte chemoattractant protein-1 by cultured human mesangial cells. J. Immunol. 148:2148-2153.

14. Hora, K., et al. 1992. Receptors for IgG complexes activate synthesis of monocyte chemoattractant peptide 1 and colony-stimulating factor 1 . Proc. Natl. Acad. Sci. USA. 89:1745-1749.

15. Schmouder, R.L., Strieter, R.M., and Kunkel, S.L. 1993. Interferongamma regulation of human renal cortical epithelial cell-derived monocyte chemotatic peptide-1. Kidney Int. 44:43-49.

16. Stahl, R.A., et al. 1993. Increased expression of monocyte chemoattractant protein-1 in anti-thymocyte antibody-induced glomerulonephritis. Kidney Int. 44:1036-1047.

17. Safirstein, R., et al. 1991. Expression of cytokine-like genes JE and KC is increased during renal ischemia. Am. J. Physiol. 261:F1095-F1101.

18. Diamond, J.R., Kees-Folts, D., Ding, G., Frye, J.E., and Restrepo, N.C. 1994. Macrophages, monocyte chemoattractant peptide-1, and TGF- $\beta 1$ in experimental hydronephrosis. Am. J. Physiol. 266:F926-F933.

19. Eddy, A.A., and Giachelli, C.M. 1995. Renal expression of genes that promote interstitial inflammation and fibrosis in rats with protein-overload proteinuria. Kidney Int. 47:1546-1557.

20. Pichler, R., et al. 1994. Tubulointerstitial disease in glomerulonephritis. Potential role of osteopontin (uropontin). Am. J. Pathol. 144:915-926.

21. Deckers, J.G., Van der Woude, F.J., Van der Kooij, S.W., and Daha, M.R. 1998. Synergistic effect of IL-1-alpha, IFN-gamma and TNF-alpha on RANTES production by human renal tubular epithelial cells in vitro. $J$. Am. Soc. Nephrol. 9:194-202.

22. Rovin, B.H., Doe, N., and Tan, L.C. 1996. Monocyte chemoattractant 
protein-1 levels in patients with glomerular disease. Am. J. Kidney Dis. 27:640-646.

23. Boring, L., et al. 1997. Impaired monocyte migration and reduced type 1 (Th1) cytokine responses in C-C chemokine receptor 2 knockout mice. J. Clin. Invest. 100:2552-2561.

24. Kurihara, T., Warr, G., Loy, J., and Bravo, R. 1997. Defects in macrophage recruitment and host defense in mice lacking the CCR2 chemokine receptor. J. Exp. Med. 186:1757-1762.

25. Kuziel, W.A., et al. 1997. Severe reduction in leukocyte adhesion and monocyte extravasation in mice deficient in CC chemokine receptor 2. Proc. Natl. Acad. Sci. USA. 94:12053-12058.

26. Tipping, P.G., Huang, X.R., Berndt, M.C., and Holdsworth, S.R. 1994. A role for P-selectin in complement-independent neutrophil-mediated glomerular injury. Kidney Int. 46:79-88.

27. Moore, K.J., Naito, T., Martin, C., and Kelley, V.R. 1996. Enhanced response of macrophages to CSF- 1 in autoimmune mice. A gene transfer strategy. J. Immunol. 157:433-440.

28. Bloom, R.D., Florquin, S., and Kelley, V.R. 1993. Colony stimulating factor-1 in the induction of lupus nephritis. Kidney Int 43:1000-1009.

29. Lloyd, C.M., et al. 1997. RANTES and monocyte chemoattractant protein-1 (MCP-1) play an important role in the inflammatory phase of crescentic nephritis, but only MCP-1 is involved in crescent formation and interstitial fibrosis. J. Exp. Med. 185:1371-1380.

30. Wuthrich, R.P., et al. 1990. MHC class II, antigen presentation and tumour necrosis factor in renal tubular epithelial cells. Kidney Int 37:783-792.

31. Vermes, I., Haanen, C., Steffens-Nakken, H., and Reutelingsperger, C. 1995. A novel assay for apoptosis. Flow cytometric detection of phosphatidylserine expression on early apoptotic cells using fluorescein labelled Annexin V. J. Immunol. Methods. 194:39-51.

32. Gunn, M.D., Nelken, N.A., Liao, X., and Williams, L.T. 1997. Monocyte chemoattractant protein- 1 is sufficient for the chemotaxis of monocytes and lymphocytes in transgenic mice but requires an additional stimulus for inflammatory activation. J. Immunol. 158:376-383.

33. Grewal, I.S., et al. 1997. Transgenic monocyte chemoattractant protein1 (MCP-1) in pancreatic islets produces monocyte-rich insulitis without diabetes: abrogation by a second transgene expressing systemic MCP-1. J. Immunol. 159:401-408.

34. Leenen, P.J., De Bruijn, M.F., Voerman, J.S., Campbell, P.A., and Van Ewijk, W. 1994. Markers of mouse macrophage development detected by monoclonal antibodies. J. Immunol. Methods. 174:5-19.

35. Natori, Y., Sekiguchi, M., Ou, Z., and Natori, Y. 1997. Gene expression of CC chemokines in experimental crescentic glomerulonephritis (CGN). Clin. Exp. Immunol. 109:143-148.

36. Franci, C., Wong, L.M., Van, D.J. Proost, P., and Charo, I.F. 1995. Monocyte chemoattractant protein-3, but not monocyte chemoattractant protein-2, is a functional ligand of the human monocyte chemoattractant protein-1 receptor. J. Immunol. 154:6511-6517.

37. Sarafi, M.N., Garcia-Zepeda, E.A., MacLean, J.A., Charo, I.F., and Luster, A.D. 1997. Murine monocyte chemoattractant protein (MCP)-5: a novel $\mathrm{CC}$ chemokine that is a structural and functional homologue of human MCP-1. J. Exp. Med. 185:99-109.

38. Tang, W.W., Qi, M., and Warren, J.S. 1996. Monocyte chemoattractant protein-1 mediates glomerular macrophage infiltration in anti-GBM Ab GN. Kidney Int. 50:665-671.

39. Wada, T., et al. 1996. Intervention of crescentic glomerulonephritis by antibodies to monocyte chemotactic and activating factor (MCAF/MCP1). FASEB. J. 10:1418-1425.

40. Fujinaka, H, et al. 1997. Suppression of anti-glomerular basement membrane nephritis by administration of anti-monocyte chemoattractant protein-1 antibody in WKY rats. J. Am. Soc. Nephrol. 8:1174-1178.

41. Doerschuk, C.M., et al. 1996. The role of P-selectin and ICAM-1 in acute lung injury as determined using blocking antibodies and mutant mice. J. Immunol. 157:4609-4614.

42. Ma, J., et al. 1996. Autoimmune lpr/lpr mice deficient in CD40 ligand: spontaneous Ig class switching with dichotomy of autoantibody responses. J. Immunol. 157:417-426.

43. Early, G.S., Zhao, W., and Burns, C.M. 1996. Anti-CD40 ligand antibody treatment prevents the development of lupus-like nephritis in a subset of New Zealand black x New Zealand white mice. Response correlates with the absence of an anti-antibody response. J. Immunol. 157:3159-3164 